\title{
Large representation Polyakov loop in hot Yang-Mills theory
}

\author{
Gianluca Grignani \\ Dipartimento di Fisica, Università di Perugia and I.N.F.N. Sezione di Perugia, Via Pascoli, \\ 06123 Perugia, Italy \\ E-mail: grignaniepg.infn.it \\ Joanna Karczmarek, Gordon W. Semenoff* \\ Department of Physics and Astronomy, University of British Columbia, 6224 Agricultural Road, \\ Vancouver, British Columbia, Canada V6T $1 Z 1$ \\ E-mail: joanna, gordonws@phas.ubc.ca
}

\begin{abstract}
We review the argument that there is a quantum phase transition in the expectation value of the Polyakov loop operator in a large representation of the gauge group. The transition occurs as the size of the representation is increased. Our analysis applies to the 't Hooft limit of four dimensional Yang-Mills theory with adjoint or other center neutral matter fields, including the case of $\mathscr{N}=4$ Yang-Mills theory on a spatial $S^{3}$. We speculate that this transition is reflected in the D-branes which are the string theory duals of giant loops.
\end{abstract}

International Workshop on QCD Greens Functions, Confinement, and Phenomenology - QCD-TNT09 September $07-112009$

ECT Trento, Italy

*Speaker. 


\section{Introduction}

A beautiful picture of the quark deconfinement phase transition of finite temperature YangMills theory has emerged in the context of AdS/CFT duality [1]. The gravitational dual of this phenomenon is the collapse of hot Anti-de Sitter space to an anti-de Sitter-Schwarzchild black hole. Unlike asymptotically flat space, where a thermodynamic state is unstable to black hole collapse no matter what the temperature, at sufficiently low temperatures, anti-de Sitter space is thought to be stable. It contains a hot gas of gravitons and possibly other particles. Moreover, when it is heated to a certain critical temperature, the hot gas eventually undergoes gravitational collapse to a spacetime which is an anti-de Sitter black hole. The temperature and entropy of the resulting spacetime are then identified with the Hawking temperature and Beckenstein-Hawking entropy of the black hole. The phase transition between the two phases is first order and is called the Hawking-Page transition [2]. It is thought to be the gravity dual of the deconfinement transition of finite temperature Yang-Mills theory.

In the strict sense, deconfinement in AdS/CFT refers to the phenomenon in $\mathscr{N}=4$ supersymmetric Yang-Mills theory quantized on a spatial three-sphere, $S^{3}$. AdS/CFT duality identifies this theory with IIB superstring theory on a background $A d S_{5} \times S^{5}$ spacetime. The latter reduces to type IIB supergravity on an asymptotically $A d S_{5} \times S^{5}$ spacetime only when the string coupling constant, which governs the breaking and joining of strings is small and in a low energy limit, where energies are small compared to the curvature of the background. In the gauge theory, these limits correspond to the large $N$ 't Hooft limit, $N \rightarrow \infty$ with $\lambda=g_{Y M}^{2} N$ held fixed, where $g_{Y M}$ is the Yang-Mills coupling constant, and then a subsequent large 't Hooft coupling, $\lambda \rightarrow \infty$ limit. By studying the Hawking page phase transition, one is studying deconfinement in the strong coupling limit of the gauge theory. The large $N$ limit is important here since, due to the finite spatial volume, a phase transition can only occur when $N$ is infinite. However one expects that the phase transition would persist if one relaxes the large $\lambda$ limit. On the string side of the duality, very little can be done away from the large $\lambda$ supergravity limit. The only other region where analytic computation is possible is in the weak coupling, small $\lambda$ limit. That limit has recently been examined and found to have a large $N$ phase transition which is identified as deconfinement [3]-[4]. It is conjectured that the two limits are connected by a line of deconfining phase transitions which stretches across the intermediate $\lambda$ regime.

In this Article, we shall review some of our recent work on the properties of the behavior of some particular Polyakov loop expectation values in deconfined phase of the gauge theory [5]. This work was motivated by an observation in string theory concerning the gravity dual of the Polyakov loops that we will discuss [6]. We will elaborate on this motivation in later Sections.

\section{Confinement-deconfinement and the Polyakov loop}

Let us begin by reviewing the role of the Polyakov loop operator as an order parameter for confinement in an adjoint gauge field theory [7]-[8]. The Polyakov loop,

$$
\left\langle\operatorname{Tr} \mathscr{P} e^{i \int_{0}^{\beta} d \tau A_{0}(\tau, \vec{x})}\right\rangle=\frac{\int\left[d A_{\mu} \ldots\right] e^{-\int \frac{1}{2 g_{Y M}^{2}} \operatorname{Tr} F_{\mu \nu}^{2}+\ldots} \operatorname{Tr} \mathscr{P} e^{i \oint_{0}^{\beta} d \tau A_{0}(\tau, \vec{x})}}{\int\left[d A_{\mu} \ldots\right] e^{-\int \frac{1}{2 g_{Y M}^{2}} \operatorname{Tr} F_{\mu \nu}^{2}+\ldots}}
$$


measures the trace of the holonomy of the gauge group on the Euclidean time circle. In pure YangMills theory, or a theory such as $\mathscr{N}=4$ super Yang-Mills theory where all matter fields transform in the adjoint representation of the gauge group, the Euclidean path integral has a center symmetry. It arises from gauge transformations which, in such a theory need not be periodic in Euclidean time, they need only preserve the periodicity of the local fields. As such, they can obey a boundary condition

$$
g(\tau+\beta, \vec{x})=\operatorname{cg}(\tau, \vec{x})
$$

where $c$ where $c=e^{2 \pi i / N}$ is the generator of the $Z_{N}$ center of the $S U(N)$ gauge group. Because $c$ commutes with everything, all local operators which transform in the adjoint representation, for example $F_{\mu v}(\tau, \vec{x}) \rightarrow g(\tau, \vec{x}) F_{\mu v}(\tau, \vec{x}) g^{\dagger}(\tau, \vec{x})$, remain periodic after the gauge transform. However, the Polyakov loop transforms as

$$
\operatorname{Tr} \mathscr{P} e^{i \int_{0}^{\beta} d \tau A_{0}(\tau, \vec{x})} \rightarrow c \operatorname{Tr} \mathscr{P} e^{i \int_{0}^{\beta} d \tau A_{0}(\tau, \vec{x})}
$$

The center transformation appears as a global symmetry of the Euclidean path integral and it acts on Polyakov loops. When it is a good symmetry, any combination of Polyakov loops with nonvanishing center-charge must have vanishing expectation value. This is interpreted as confinement. When the center symmetry is spontaneously broken, the expectation value can be non-zero. This is interpreted as deconfinement. The representation of center symmetry has been used to study the deconfining phase transition of Yang-Mills theory [9]-[12].

The expectation value of the Polyakov loop operator is related to the free energy of Yang-Mills theory with a classical color source inserted. If one quantizes Yang-Mills theory with the constraint that a non-dynamical color charge is located at point $\vec{x}$, the free energy of the system $\Gamma$ is obtained from the Euclidean path integral in (2.1).

$$
e^{-\beta \Gamma}=\left\langle\operatorname{Tr} \mathscr{P} e^{i \int_{0}^{\beta} d \tau A_{0}(\tau, \vec{x})}\right\rangle
$$

$\Gamma$ is the energy that is needed to insert the external charge. When the expectation value is zero, as in the confining phase, the energy is infinite. When the expectation value is non-zero, the energy is finite.

\section{Effective field theory}

In recent work, the weak coupling limit of both Yang-Mills theory and $\mathscr{N}=4$ super YangMills theory have been studied for the case where the space-time is $S^{3} \times S^{1}$ [3]-[4]. This is an interesting case in that, at weak coupling, the spectrum of the vector gluon fields is completely gapped. If they are conformally coupled to the curvature of the $S^{3}$, the scalar fields of $\mathscr{N}=4$ theory are also gapped, as are the fermionic quarks. Then, in the regime where the temperature is much less than the gap, $T=\frac{1}{\beta}<<1$ (we choose the $S^{3}$ to have unit radius), an effective field theory technique can be used to find an effective action for the Polyakov loop. All of the propagating degrees of freedom can be integrated out, leaving an effective field theory for the Polyakov loop operator itself. The expectation value of the (appropriately renormalized) loop operator can then be computed using this effective field theory which is a unitary one-matrix model,

$$
\left\langle\operatorname{Tr} \mathscr{P} e^{i \int_{0}^{\beta} d \tau A_{0}(\tau, x)}\right\rangle=\frac{\int d U e^{-S_{\text {eff }}[U]} \operatorname{Tr} U}{\int d U e^{-S_{\text {eff }}[U]}}
$$


where, at one-loop order, the effective action is

$$
S_{\mathrm{eff}}[U]=-\sum_{n=1}^{\infty}\left[z_{B}\left(x^{n}\right)+(-1)^{n+1} z_{F}\left(x^{n}\right)\right] \frac{\left|\operatorname{Tr} U^{n}\right|^{2}}{n}
$$

where

$$
x=e^{-\frac{\beta}{R}}, z_{B}(x)=\frac{6 x+12 x^{2}-x^{3}}{(1-x)^{3}}, z_{F}(x)=\frac{16 x^{\frac{3}{2}}}{(1-x)^{3}}
$$

Here, we have presented the action for $\mathscr{N}=4$ Yang-Mills theory. The one for pure Yang-Mills theory is similar and is presented in Ref. [4]. $S_{\text {eff }}$ in (3.2) does not depend on the 't Hooft coupling $\lambda$. This is due to the fact that it is the one-loop approximation. Dependence on $\lambda$ starts at two loops. For pure Yang-Mills theory, it has been partially found up to order two loops [13] where the first order nature of the phase transition is confirmed.

The effective action $S_{\text {eff }}$ inherits symmetries from its parent theory:

- Gauge invariance,

$$
S_{\text {eff }}[U]=S_{\text {eff }}\left[V U V^{-1}\right]
$$

Note that the effective action (3.2) depends only on invariant quantities $\operatorname{Tr} U^{n}$.

- Center symmetry

$$
S_{\text {eff }}[U]=S_{\text {eff }}[c U]
$$

Note that in (3.2), the effective action depends on $U$ only in the center symmetric combinations $\operatorname{Tr} U^{n} \operatorname{Tr} U^{\dagger n}$.

- Further, the action is of order $N^{2}, S_{\text {eff }}[U=1] \sim N^{2}$.

Gauge invariance (3.4) allows one to diagonalize the unitary matrices to form a model of the eigenvalues. It turns out that this model can be solved by a saddle-point method in the large $N$ limit and it is found that it has a phase transition. The expectation value of the Polyakov loop vanishes in the low temperature phase and it is nonzero in the high temperature phase. This is interpreted as a deconfinement transition which occurs even at weak coupling. For the effective action (3.2), the phase transition occurs at $T_{C} \simeq 0.38$ which is marginal to the regime $T<<1$. We will assume that it is within the range of validity of the effective field theory technique. In the following we will explore the deconfined phase. We will assume that we are at temperatures just above the critical one and we will assume that the effective matrix model gives an accurate description of the physics there.

The analysis of unitary matrix models and the existence of a phase transition of the type that we are discussing here has a long history dating back to the seminal work of Gross and Witten [14].

\section{Higher representations}

The unitary matrix model can be used to calculate the expectation value of the Polyakov loop operator in any irreducible representation $R$ of the $S U(N)$ gauge group,

$$
\left\langle\operatorname{Tr}_{R} U(x)\right\rangle=\frac{\int[d U] e^{-S_{\text {eff }}[U]} \operatorname{Tr}_{R} U}{\int[d U] e^{-S_{\text {eff }}[U]}}
$$


where $\operatorname{Tr}_{R} U$ is called the character. We shall show in the following that (4.1) can have interesting behavior which depends on the size and nature of the representation. Two types of representation are easy to analyze: the completely symmetric representations $\mathscr{S}_{k}$ whose Young tableaux are a single row with $k$ boxes and completely antisymmetric representations $\mathscr{A}_{k}$ whose Young tableaux are a single column with $k$ boxes. We shall consider large values of $k$ so that the ratio $\frac{k}{N}$ remains finite as $N \rightarrow \infty$ and we will study the behavior of these representations as $\frac{k}{N}$ is varied.

Note that we have not normalized the Polyakov loop, as one would normally do by dividing by a factor of the dimension of the representation. Our reason for not doing so is to be able to compare our results directly with holographic duality where the appropriate operator is the un-normalized one. This immediately introduces the interesting possibility that the expectation value is bigger than one - for example if the averaging over $U$ were concentrated at the unit matrix - the expectation value would simply be equal to the dimension of the representation. The free energy would be negative, indicating that the system would "attract" the heavy quark. This apparent attraction is not dynamical, it is statistical, simply due to the increase in entropy from the multiplicity of states of the quark.

The center charge of a representation is equal to the number of boxes in the Young Tableau corresponding to that representation, modulo $N$. Thus, both representations $\mathscr{S}_{k}$ and $\mathscr{A}_{k}$ have center charge $k \bmod N$. The expectation value (4.1) is therefore expected to vanish in the confined phase when this charge is non-zero. On the other hand, the expectation value can be non-zero in the deconfined phase.

If the matrix $U=\operatorname{diag}\left[e^{i \phi_{1}}, \ldots, e^{i \phi_{N}}\right]$ were diagonal, the permutation symmetry can be used to order the eigenvalues in a completely symmetric or completely antisymmetric representation so that they occur in order of non-decreasing index:

$$
\operatorname{Tr}_{\mathscr{S}_{k}} U=\sum_{a_{1} \leq a_{2} \leq \ldots \leq a_{k}} e^{i \phi_{a_{1}}} e^{i \phi_{a_{2}} \ldots} e^{i \phi_{a_{k}}}, \operatorname{Tr}_{\mathscr{A k}_{k}} U=\sum_{a_{1}<a_{2}<\ldots<a_{k}} e^{i \phi_{a_{1}}} e^{i \phi_{a_{2}} \ldots} e^{i \phi_{a_{k}}}
$$

It is convenient to obtain these expressions from generating functions

$$
\operatorname{Tr}_{\mathscr{S}_{k}} U=\oint \frac{d t}{2 \pi i t^{k+1}} \prod_{a=1}^{N} \frac{1}{1-t e^{i \phi_{a}}}, \operatorname{Tr}_{\mathscr{A}_{k}} U=\oint \frac{d t}{2 \pi i t^{k+1}} \prod_{a=1}^{N}\left(1+t e^{i \phi_{a}}\right)
$$

where the contour integral projects onto the term in a Taylor expansion of the integrand which contains $k$ eigenvalues. The contour in the integral over $t$ encircles the origin. It can be moved away from the origin if it does not cross singularities of the integrand. In the case of the antisymmetric representation (4.3) when $N$ is finite, the integrand is a polynomial and the contour can be moved anywhere. For the symmetric representation (4.3) it should remain within the unit circle.

These can be written as the covariant expressions for the free energies,

$$
\begin{array}{r}
\beta \Gamma_{\mathscr{S}_{k}} \equiv-\frac{1}{N} \ln \left\langle\operatorname{Tr}_{\mathscr{S}_{k}} U\right\rangle=-\frac{1}{N} \ln \frac{1}{2 \pi i} \oint d t \frac{1}{t^{k+1}}\langle\exp [-\operatorname{Tr} \ln (1-t U)]\rangle, \\
\beta \Gamma_{\mathscr{A}_{k}} \equiv-\frac{1}{N} \ln \left\langle\operatorname{Tr}_{\mathscr{A}_{k}} U\right\rangle=-\frac{1}{N} \ln \frac{1}{2 \pi i} \oint d t \frac{1}{t^{k+1}}\langle\exp [\operatorname{Tr} \ln (1+t U)]\rangle
\end{array}
$$

The characters in (4.4) and (4.5) have center charge $k$ and therefore they must vanish in the center symmetric confining phase. They can be non-zero in the deconfined phase. 
In the large $N$ limit, the quantities in (4.4) and (4.5) can be computed using two saddle point approximations. The first occurs while integrating over unitary matrices in (4.1). Because of the gauge symmetry, this is an eigenvalue model. The gauge symmetry can be used to diagonalize $U$. At large $N$, the eigenvalues become classical variables and their distribution is found by minimizing $S_{\text {eff }}$ plus a Jacobian from the unitary integral measure. As long as $k \ll N^{2}$, the loop operators in (4.4) do not modify the eigenvalue distribution in the leading order at large $N$. It is given by a density $\rho(\phi)$ which is $\frac{1}{N}$ times the number of eigenvalues between $\phi$ and $\phi+d \phi$ and is normalized, $\int_{-\pi}^{\pi} d \phi \rho(\phi)=1$. In the large $N$ limit the expectation values in Eq. (4.4) are computed using the eigenvalue density, ${ }^{1}$

$$
\begin{gathered}
\beta \Gamma_{\mathscr{S}_{k}}=-\frac{1}{N} \ln \frac{1}{2 \pi i} \oint d t \frac{1}{t} \exp \left(-N \int_{-\pi}^{\pi} d \phi \rho(\phi) \ln \left(1-t e^{i \phi}\right)-k \ln t\right) . \\
\beta \Gamma_{\mathscr{\mathscr { A } _ { k }}}=-\frac{1}{N} \ln \frac{1}{2 \pi i} \oint d t \frac{1}{t} \exp \left(N \int_{-\pi}^{\pi} d \phi \rho(\phi) \ln \left(1+t e^{i \phi}\right)-k \ln t\right) .
\end{gathered}
$$

The second use of a saddle-point approximation is to evaluate the integral over $t$ in (4.6) and (4.7). Let $\hat{t}$ satisfy the saddle-point equation

$$
R_{\mathscr{S}_{k}}(\hat{t}) \equiv \int_{-\pi}^{\pi} d \phi \rho(\phi) \frac{\hat{t} e^{i \phi}}{1-\hat{t} e^{i \phi}}=\frac{k}{N}, R_{\mathscr{A} k}(\hat{t}) \equiv \int_{-\pi}^{\pi} d \phi \rho(\phi) \frac{\hat{t} e^{i \phi}}{1+\hat{t} e^{i \phi}}=\frac{k}{N} .
$$

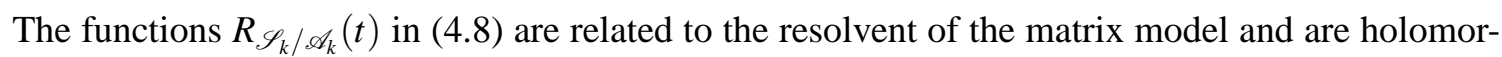
phic functions of $t$ with cut singularities on the unit circle determined by the support of $\rho(\phi)$. Once the solution $\hat{t}$ of the saddle point is determined, the free energy is given by

$$
\begin{gathered}
\beta \Gamma_{\mathscr{S}_{k}}=\int_{-\pi}^{\pi} d \phi \rho(\phi) \ln \left(1-\hat{t} e^{i \phi}\right)+\frac{k}{N} \ln \hat{t} . \\
\beta \Gamma_{\mathscr{A}_{k}}=-\int_{-\pi}^{\pi} d \phi \rho(\phi) \ln \left(1+\hat{t} e^{i \phi}\right)+\frac{k}{N} \ln \hat{t} .
\end{gathered}
$$

The generating function technique that we have used in the above is well known. See Ref. [15] for a recent application in a different context.

The reader might have the concern that the presence of the loop variable in the path integral, though it does not alter the eigenvalue distribution to the leading order $N^{0}$, it will have an effect at order $1 / N$ and a $1 / N$ correction in the order $N^{2}$ part of the action would contribute a term of order $N$ which competes with the free energy which we are computing. To see why this is not a problem, consider the free energy in the large $N$ limit is given by

$$
N \beta \Gamma=\inf _{(\rho, t)}\left[N^{2} S[\rho]+\lambda \int \rho-\lambda+N \int \rho \ln \left(1-t e^{i \phi}\right)+k \ln t\right]-\inf _{\rho}\left[N^{2} S[\rho]+\lambda \int \rho-\lambda\right]
$$

where $S[\rho]$ is the effective action consisting of $S_{\text {eff }}$ plus a contribution from the integration measure. The saddle-point equations are

$$
\frac{\delta S}{\delta \rho}+\frac{1}{N} \ln \left(1-t e^{i \phi}\right)+\frac{\lambda}{N^{2}}=0, \quad \int \rho=1, \quad \int \rho \frac{t e^{i \phi}}{1-t e^{i \phi}}=\frac{k}{N} \frac{1}{t}
$$

\footnotetext{
${ }^{1}$ We will argue that using the leading order $N^{0}$ density $\rho(\phi)$ in (4.6) is sufficient to obtain $\beta \Gamma_{\mathscr{S}_{k} / \mathscr{A}_{k}}$ to leading order $N^{0}$ accuracy.
} 
for the first infimum and

$$
\frac{\delta S}{\delta \rho}+\frac{\lambda}{N^{2}}=0, \quad \int \rho=1
$$

for the second infimum. The eigenvalue density which satisfies (4.13) is $\hat{\rho}_{0}$. Then the density which satisfies (4.12) differs from it by a correction of order $\frac{1}{N}, \hat{\rho}_{0}+\frac{1}{N} \hat{\rho}_{1}$. However, since $\hat{\rho}_{0}$ satisfies (4.13), it is easy to see, that if we are interested in $N \beta \Gamma$ only to accuracy of order $N$, we can get simply use $\hat{\rho}_{0}$ in the equation which determines $\hat{t}$ and, to the same accuracy (where we trust the order $N$ but not the order $N^{0}$ contribution), in the expression for $N \beta \Gamma$ in (4.11). This justifies our use of the "probe approximation" where we use the eigenvalue distribution of the effective unitary matrix model to compute the generating functions in (4.6) to (4.10). We note that a similar probe approximation is made when analyzing the dual objects on the string theory side of the AdS/CFT correspondence.

Before we proceed further, let us consider a simple example, the confined phase. Center symmetry is an invariance under a simultaneous translation of all eigenvalues $\phi_{a} \rightarrow \phi_{a}+2 \pi / N$. In the center-symmetric confined phase, the distribution is translation invariant, eigenvalues are uniformly distributed on the unit circle and

$$
\rho_{\text {conf }}=\frac{1}{2 \pi}
$$

In the de-confined phase, on the other hand, the eigenvalues would have a non-constant distribution. Let us put off discussing the de-confined phase until later.

With the confining eigenvalue distribution (4.14) we can integrate over $\phi$ in the saddle-point equations (4.8),

$$
R_{\mathscr{S}_{k}}(\hat{t})=\left\{\begin{array}{cc}
0 & |\hat{t}|<1 \\
-1 & |\hat{t}|>1
\end{array}=\frac{k}{N}, R_{\mathscr{A}_{k}}(\hat{t})=\left\{\begin{array}{ll}
0 & |\hat{t}|<1 \\
1 & |\hat{t}|>1
\end{array}=\frac{k}{N}\right.\right.
$$

Similarly, and consistent with this, we can integrate the free energies in (4.9) and (4.10),

$$
\beta \Gamma_{\mathscr{S}_{k}}=\left\{\begin{array}{cc}
\frac{k}{N} \ln \hat{t} & |t|<1 \\
i \pi+\left(1+\frac{k}{N}\right) \ln \hat{t} & |t|>1
\end{array}, \beta \Gamma_{\mathscr{A}_{k}}=\left\{\begin{array}{cc}
\frac{k}{N} \ln \hat{t} & |t|<1 \\
\left(\frac{k}{N}-1\right) \ln \hat{t} & |t|>1
\end{array}\right.\right.
$$

In the case of the symmetric representation, the saddle-point equation (4.15) has a solution only when $\frac{k}{N}=0$. We interpret the absence of a solution when $\frac{k}{N} \neq 0$ as meaning that the expectation value vanishes. Certainly, if there is no saddle-point of a periodic function of a variable $\phi$, the integration is not dominated by any particular value of $\phi$ and $\phi$ must be integrated over its entire range. This would average the expectation value of any operator with non-zero center charge to zero. It is in the other case, when there is a saddle point, where the large $N$ limit forces one to evaluate the integrand at the saddle point and the expectation value is generically non-zero. Further, we see that the free energy has an imaginary part when $|\hat{t}|>1$ which indicates an instability. This apparent pathology is consistent with the observation after (4.3) that the integration contour should remain inside the unit circle. We shall henceforth ignore the region. ${ }^{2}$

\footnotetext{
${ }^{2}$ We do later consider the analytic continuation of the free energy and the solution of the saddle point equation from the region $|t|<1$ to the entire complex plane.
} 
Similarly, for the anti-symmetric representation, the saddle-point equation (4.15) has a solution only when either $\frac{k}{N}=0$ or $\frac{k}{N}=1$, the two cases where the antisymmetric representation is center neutral. This is also interpreted as confinement, the expectation value vanishes in all other cases. Note that it has an expected $k \rightarrow N-k$ duality, though it comes from interchanging two saddle points, one with $|\hat{t}|<1$ and one with $|\hat{t}|>1$. Neither of these saddle-points alone exhibit this duality.

We will review the computation of the higher representation Polyakov loops with more complicated eigenvalue distributions. Before that, in the next Section, we pause to review some of the motivation for considering them. This motivation derives from the analysis of large representation Wilson loops and the string theory dual objects in zero temperature de-confined $\mathscr{N}=4$ Yang-Mills theory.

\section{Giant Wilson loops}

In the duality between gauge field theory and string theory, the expectation value of the Wilson loop is normally thought to correspond to an open fundamental string amplitude in string theory. This has been made precise for the Maldacena-Wilson loop [16] which differs from the Polyakov loop (4.1) in that it contains the scalar field of the $\mathscr{N}=4$ theory as well as the gauge field,

$$
W_{\mathrm{M}}[C]=\operatorname{Tr} \mathscr{P} e^{\oint_{C} d \tau\left(i A_{\mu}(x) \dot{x}^{\mu}(\tau)+\phi^{I}(x) \theta^{I}|\dot{x}(\tau)|\right)}
$$

where $x^{\mu}(\tau)$ parametrizes a closed curve $C$. $\phi^{I}(x), I=1, \ldots, 6$ are the scalar quark fields of $\mathscr{N}=4$ super Yang-Mills theory and $\theta^{I}$ is a (not necessarily $\tau$-independent) unit 6-vector.

In that case, the boundary of the fundamental string worldsheet is located on the loop contour $C$ placed at the asymptotic boundary of $A d S_{5}$. The Maldacena-Wilson loop could be made to link periodic Euclidean time in the finite temperature field theory. Then its string theory dual would be a disc amplitude where the boundary of the disc is located at infinity. Whether such a disc exists depends on whether the time circle is contractable. It is not contractable in the hot AdS background, and it is contractable on the black hole background. This was pointed out by Witten as further evidence for the identification of the Hawking-Page transition with deconfinement [1].

If $C$ wraps the time circle, $W_{\mathrm{M}}[C]$ carries center charge and, like the Polyakov loop, its expectation value is governed by the realization of the center symmetry. Its expectation value is related to the holonomy of a heavy $W$-boson which would be created by Higgsing $S U(N+1)$ gauge symmetry to $S U(N) \times U(1)$ and where $\theta^{I}$ gives the orientation of the scalar condensate. One could imagine larger representation objects made from $W$-bosons, for example a bound state of a large number of the $W$-bosons which transforms in a higher representation of the gauge group.

In the zero temperature Yang-Mills theory defined on a spatial $R^{3}$, an interesting phenomenon occurs for loops in representations where the number of boxes $k$ in the Young tableau is large so that $\frac{k}{N}$ is finite in the large $N$ limit. The dual fundamental string worldsheet is replaced by a D-brane with world-volume electric flux [17].

This was found by studying highly supersymmetric $\frac{1}{2}$-BPS loops, where some results are known for all values of the coupling constant [18]. For the anti-symmetric representation, the dual is a D5-brane whose world volume is a direct product of $A d S_{2} \subset A d S_{5}$ and $S^{4} \subset S^{5}$. For a symmetric representation, it is a D3-brane with world volume $A d S_{2} \times S^{2} \subset A d S_{5}$. 
It is interesting to ask whether these D-branes exist in the finite temperature geometry where they would be dual to a gauge theory loop linking periodic Euclidean time. This question was studied by Hartnoll and Kumar [6] who searched for solutions of the appropriate Born-Infeld actions on the black hole background.

For the D5-brane wrapped on $S^{4} \subset S^{5}$ which corresponds to a totally antisymmetric representation on the gauge theory side, there seem to be solutions for any $\frac{k}{N}$ with the usual cutoff at $k=N$ dictated by the maximum size of an antisymmetric representation on the gauge theory side and a maximum radius for embedding $S^{4}$ in $S^{5}$ on the supergravity side. However, in the case of the D3brane, which should correspond to a totally symmetric representation, Hartnoll and Kumar could not find any solutions at all. This fundamental difference between the two cases is what motivated our work on the gauge theory which is summarized in Ref. [5] and which we are reviewing here.

We emphasize that in supergravity we are studying the dual of the Maldacena-Wilson loop whereas in gauge theory our analysis is limited to the Polyakov loop. Both are governed by the center symmetry and both can become non-zero at the deconfinement transition. At high temperature, due to decoupling of the scalar fields, they should become similar.

\section{Giant Polyakov loop}

Let us begin with the symmetric representation $\mathscr{S}_{k}$. We shall consider three examples of eigenvalue distributions. First, the confining phase has $\rho_{\text {conf }}=\frac{1}{2 \pi}$. $R_{\mathscr{S}_{k}}(t)$ vanishes if $|t|<1$ and is -1 if $|t|>1$. This is the expected discontinuity at the unit circle. Eq. (4.8) has solutions only when $\frac{k}{N}=0$, consistent with confinement.

As a second example consider $\rho(\phi)=\frac{1}{2 \pi}(1+2 p \cos \phi) . p=\frac{1}{N}\langle\operatorname{Tr} U\rangle=\int d \phi \rho(\phi) e^{i \phi}$ is the fundamental representation loop. Positivity of the density requires $0 \leq p \leq \frac{1}{2}$. This distribution depends on $\phi$ and therefore is deconfined. While it is not realistic for $\mathscr{N}=4$ Yang-Mills theory, it does occur in the strong-coupling phase of large $N$ 2-dimensional lattice Yang-Mills theory [14].

There is one solution of $R_{\mathscr{S}_{k}}(\hat{t})=\frac{k}{N}$ in the region $|\hat{t}|<1$ at $\hat{t}=\frac{k}{N} / p$. (If $\frac{k}{N}$ and $p$ are such that $|\hat{t}|>1$, both $R_{\mathscr{S}_{k}}$ and $\Gamma_{\mathscr{S}_{k}}$ should be extended there by analytic continuation.) The free energy is

$$
\Gamma_{\mathscr{S}_{k}}=\frac{k}{N} \ln \left[\frac{k / N}{e p}\right],
$$

where $e=2.718 \ldots \Gamma_{\mathscr{L}_{k}}$ has the interesting feature that, as $\frac{k}{N}$ is increased, it changes sign from negative to positive. This results in a phase transition which occurs when $\frac{k}{N}=\left(\frac{k}{N}\right)_{\text {crit }}=e p$. When $\frac{k}{N}<\left(\frac{k}{N}\right)_{\text {crit }}, \Gamma_{\mathscr{S}_{k}}$ is negative and the loop expectation value, $e^{-N \Gamma}$, is exponentially large. When $\frac{k}{N}>\left(\frac{k}{N}\right)_{\text {crit }}, \Gamma_{\mathscr{S}_{k}}$ is positive and the loop vanishes for $N \rightarrow \infty$. This phase transition implies that, even in the deconfined phase, sufficiently large symmetric representations are still confined.

As a check of the saddle-point approximation to the $t$-integral in this simple example, observe that, if for the moment we assume that $k$ and $N$ are finite, we can integrate (4.6) explicitly to get $e^{-N \Gamma_{\mathscr{S}_{k}}}=\frac{N^{k}}{k !} p^{k}$. Using the Stirling formula and taking $k \sim N \rightarrow \infty$ reproduces (6.1).

To see this behavior in another example, consider the semi-circle distribution which, for $|\phi|<$ $2 \arcsin \sqrt{2-2 p}$, is

$$
\rho(\phi)=\frac{\cos \frac{\phi}{2}}{\pi(2-2 p)} \sqrt{2-2 p-\sin ^{2} \frac{\phi}{2}}
$$




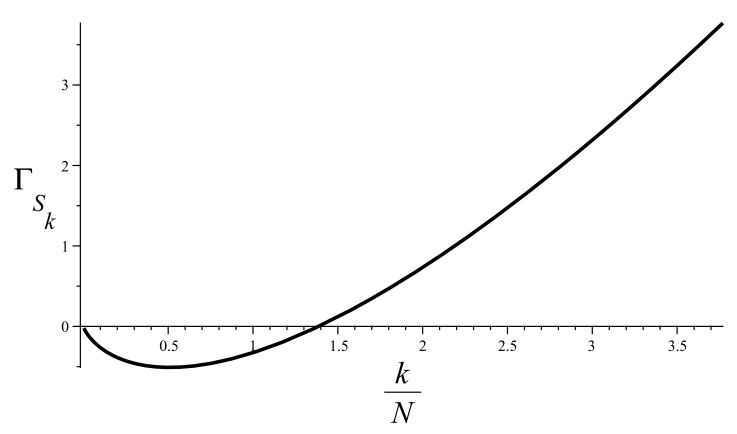

Figure 1: The free energy $\Gamma_{\mathscr{S}_{k}}(\theta)$ as a function of $\frac{k}{N}$ in the semi-circle distribution with $p=0.51$.

and which vanishes in the gap $2 \arcsin \sqrt{2-2 p} \leq|\phi| \leq \pi$. We still use the fundamental loop, $p$, as a parameter and now $\frac{1}{2} \leq p \leq 1$. This is the distribution in the weak coupling phase of 2-dimensional lattice Yang-Mills theory [14]. It is also an approximation to the deconfined distribution for weakly coupled $\mathscr{N}=4$ Yang-Mills theory [4][19]. For sufficiently weak coupling, it could be accurate near the phase transition where $p=\frac{1}{2}$. The saddle point computation can be done explicitly near $t=0$ and analytically continued. The free energy is

$$
\begin{aligned}
\Gamma_{\mathscr{S}_{k}} & =(2 \theta \cosh \theta-\sinh \theta) \frac{\sinh \theta+\sqrt{\sinh ^{2} \theta+2-2 p}}{2-2 p} \\
& -\frac{1}{2}-\ln \left[\frac{\sinh \theta+\sqrt{\sinh ^{2} \theta+2-2 p}}{2-2 p}\right],
\end{aligned}
$$

where $\theta$ is defined by $\hat{t}=e^{2 \theta}$ and is determined by the saddle-point equation

$$
\frac{k}{N}+\frac{1}{2}=\cosh \theta\left[\frac{\sinh \theta+\sqrt{\sinh ^{2} \theta+2-2 p}}{2-2 p}\right],
$$

which can be solved for $\sinh (\theta)$. The free energy is zero when $k=0$, negative for small $k$, goes to zero at a critical $\frac{k}{N}$ and is positive thereafter. This is so for any value of $p$ in the allowed range. A graph of $\Gamma_{\mathscr{S}_{k}}$ versus $\frac{k}{N}$ for $p=0.51$ is plotted in Fig. 1. With this value of $p$, the free energy becomes positive at $\theta \simeq 0.50$ which corresponds to $\frac{k}{N}$ crit. $\simeq 1.4$.

Now, we consider the antisymmetric representation. For a large class of distributions gapped around $\phi=\pi$ and with $\frac{d R_{\mathscr{A}_{k}}(\hat{t})}{d \hat{t}}>0$, which includes the semi-circle distribution (6.2), we can argue that $\Gamma_{\mathscr{A}}$ is always negative and the phase transition that we are discussing does not occur. To begin, by changing variables in (4.9), we observe that $\Gamma_{\mathscr{A}_{k}}=\Gamma_{\mathscr{A}_{N-k}}$. This symmetry is reflected in the saddle-point equation (4.8) which, using our assumption that $\rho(\phi)=\rho(-\phi)$, can be re-written as

$$
\frac{1}{2} \int_{-\pi}^{\pi} d \phi \rho(\phi) \frac{\hat{t}^{\frac{1}{2}} e^{i \frac{\phi}{2}}-\hat{t}^{-\frac{1}{2}} e^{-i \frac{\phi}{2}}}{\hat{t}^{\frac{1}{2}} e^{i \frac{\phi}{2}}+\hat{t}^{-\frac{1}{2}} e^{-i \frac{\phi}{2}}}=\frac{k}{N}-\frac{1}{2}
$$

and implies $\hat{t}(k / N)=1 / \hat{t}(1-k / N)$. The free energy,

$$
\Gamma_{\mathscr{A} k}=-\int_{-\pi}^{\pi} d \phi \rho(\phi) \ln \left(\hat{t}^{\frac{1}{2}} e^{i \frac{\phi}{2}}+\hat{t}^{-\frac{1}{2}} e^{-i \frac{\phi}{2}}\right)+\left(\frac{k}{N}-\frac{1}{2}\right) \ln \hat{t}
$$


is symmetric under $\frac{k}{N} \rightarrow 1-\frac{k}{N}$. Moreover, with a gapped distribution, $\rho(\pi)=0$, and the integral in (6.5) is continuous at $\hat{t}=1$. From $\frac{d R_{d d_{k}}(\hat{t})}{d \hat{t}}>0, \hat{t}(k)$ is monotone, and one can see in (6.5) that $\hat{t}=0$ corresponds to $\frac{k}{N}=0, \hat{t}=\infty$ to $\frac{k}{N}=1$ and $\hat{t}=1$ to $\frac{k}{N}=\frac{1}{2}$. Furthermore, since $\frac{d \Gamma_{\mathscr{d}_{k}}}{d(k / N)}=\ln \hat{t}(k)$, $\frac{d^{2} \Gamma_{\mathscr{A}_{k}}}{d(k / N)^{2}}>0$, thus $\Gamma_{\mathscr{A} k}$ is a convex function which decreases from 0 to a negative minimum as $\frac{k}{N}$ goes from 0 to $\frac{1}{2}$ and then increases back to zero when $\frac{k}{N}$ goes from $\frac{1}{2}$ to 1 . $\Gamma_{\mathscr{A}}$ does not become positive and there is no phase transition of the kind that we found for symmetric representations. When the distribution is ungapped, or when $\frac{d R_{\alpha_{k}}(\hat{t})}{d t}$ becomes negative (for example when $p<0$ ), interesting behavior can occur, discussion of which we put off to a later time.

We have found a difference between the symmetric and antisymmetric representation Polyakov loops in the gauge theory which is qualitatively similar to the one found by Hartnoll and Kumar [6] for the dual objects in supergravity: the antisymmetric loop is non-zero in the deconfined phase for all allowed $\frac{k}{N}$ and the dual D5-brane exists whereas the gauge theory symmetric representation loop has a phase transition at a critical value of $\frac{k}{N}$. The numerical search for the dual D3-brane in [6] combined with analytic arguments at large values of the parameter $\kappa=\frac{\sqrt{\lambda}}{4} \frac{k}{N}$ found no solution at all. We have also carried out a further numerical search for the D3-brane, which was partially reported in [5]. We explored the region of small $\kappa$ and found no evidence for a solution except for extremely small values of $\kappa$ which we cannot rule out. If we assume that there is no solution and that this means that the expectation value of the gauge theory quantity vanishes, it indicates that, even in the deconfined phase, the gauge theory is still confining for large charge symmetric representation sources. Of course, coming from the string theory, this is accurate only for large $\lambda$. The conclusion is that the critical value of $\frac{k}{N}$ becomes coupling constant dependent and the function $\left[\frac{k}{N}\right]_{\text {crit. }}(T, \lambda)$ goes to zero faster than $\frac{4}{\sqrt{\lambda}}$ as $\lambda \rightarrow \infty$.

We note that a phase transition of similar nature, but in a somewhat different context of branes with large angular momentum has recently been discussed [20].

The authors acknowledge hospitality of the Galileo Galilei Institute, Aspen Center for Physics and Perimeter Institute. This work is supported in part by NSERC of Canada and the INFN of Italy.

\section{References}

[1] E. Witten, "Anti-de Sitter space, thermal phase transition, and confinement in gauge theories," Adv. Theor. Math. Phys. 2, 505 (1998) [arXiv:hep-th/9803131].

[2] S. W. Hawking and D. N. Page, "Thermodynamics Of Black Holes In Anti-De Sitter Space," Commun. Math. Phys. 87, 577 (1983).

[3] B. Sundborg, "The Hagedorn transition, deconfinement and N = 4 SYM theory," Nucl. Phys. B 573, 349 (2000) [arXiv:hep-th/9908001].

[4] O. Aharony, J. Marsano, S. Minwalla, K. Papadodimas and M. Van Raamsdonk, "The Hagedorn / deconfinement phase transition in weakly coupled large N gauge theories," Adv. Theor. Math. Phys. 8, 603 (2004) [arXiv:hep-th/0310285].

[5] G. Grignani, J. L. Karczmarek and G. W. Semenoff, "Hot Giant Loop Holography,” arXiv:0904.3750 [hep-th].

[6] S. A. Hartnoll and S. Prem Kumar, "Multiply wound Polyakov loops at strong coupling," Phys. Rev. D 74, 026001 (2006) [arXiv:hep-th/0603190]. 
[7] A. M. Polyakov, “Thermal Properties Of Gauge Fields And Quark Liberation,” Phys. Lett. B 72, 477 (1978).

[8] L. Susskind, “Lattice Models Of Quark Confinement At High Temperature,” Phys. Rev. D 20, 2610 (1979).

[9] L. D. McLerran and B. Svetitsky, “A Monte Carlo Study Of SU(2) Yang-Mills Theory At Finite Temperature,” Phys. Lett. B 98, 195 (1981);

[10] B. Svetitsky and L. G. Yaffe, "Critical Behavior At Finite Temperature Confinement Transitions," Nucl. Phys. B 210, 423 (1982);

[11] L. G. Yaffe and B. Svetitsky, "First Order Phase Transition In The SU(3) Gauge Theory At Finite Temperature," Phys. Rev. D 26, 963 (1982);

[12] A. Dumitru, Y. Hatta, J. Lenaghan, K. Orginos and R. D. Pisarski, "Deconfining phase transition as a matrix model of renormalized Polyakov loops,” Phys. Rev. D 70, 034511 (2004) [arXiv:hep-th/0311223].

[13] O. Aharony, J. Marsano and M. Van Raamsdonk, "Two loop partition function for large N pure Yang-Mills theory on a small S**3,” Phys. Rev. D 74, 105012 (2006) [arXiv:hep-th/0608156].

[14] D. J. Gross and E. Witten, "Possible Third Order Phase Transition In The Large N Lattice Gauge Theory,” Phys. Rev. D 21 (1980) 446.

[15] S. A. Hartnoll and S. P. Kumar, "Higher rank Wilson loops from a matrix model," JHEP 0608, 026 (2006) [arXiv:hep-th/0605027].

[16] J. M. Maldacena, “Wilson loops in large N field theories,” Phys. Rev. Lett. 80, 4859 (1998) [arXiv:hep-th/9803002].

[17] N. Drukker and B. Fiol, "All-genus calculation of Wilson loops using D-branes," JHEP 0502, 010 (2005) [arXiv:hep-th/0501109].

S. Yamaguchi, “Bubbling geometries for half BPS Wilson lines,” Int. J. Mod. Phys. A 22, 1353 (2007) [arXiv:hep-th/0601089];

S. Yamaguchi, "Wilson loops of anti-symmetric representation and D5-branes," JHEP 0605, 037 (2006) [arXiv:hep-th/0603208].

J. Gomis and F. Passerini, “Holographic Wilson loops,” JHEP 0608, 074 (2006) [arXiv:hep-th/0604007];

J. Gomis and F. Passerini, “Wilson loops as D3-branes,” JHEP 0701, 097 (2007) [arXiv:hep-th/0612022];

J. Gomis, S. Matsuura, T. Okuda and D. Trancanelli, "Wilson loop correlators at strong coupling: from matrices to bubbling geometries,” JHEP 0808, 068 (2008) [arXiv:0807.3330 [hep-th]].

K. Okuyama and G. W. Semenoff, "Wilson loops in N = 4 SYM and fermion droplets," JHEP 0606, 057 (2006) [arXiv:hep-th/0604209];

[18] J. K. Erickson, G. W. Semenoff and K. Zarembo, "Wilson loops in N = 4 supersymmetric Yang-Mills theory," Nucl. Phys. B 582, 155 (2000) [arXiv:hep-th/0003055]; V. Pestun, "Localization of gauge theory on a four-sphere and supersymmetric Wilson loops," arXiv:0712.2824 [hep-th].

[19] J. Jurkiewicz and K. Zalewski, "Vacuum Structure Of The U(N $\rightarrow$ Infinity) Gauge Theory On A Two-Dimensional Lattice For A Broad Class Of Variant Actions,” Nucl. Phys. B 220, 167 (1983).

[20] D. Gang, J. S. Park and S. Yamaguchi, “Operator with large spin and spinning D3-brane,” JHEP 0911, 024 (2009) [arXiv:0908.3938 [hep-th]]. 